\title{
ON THE ANTECEDENTS OF PALMAR HYPERHIDROSIS
}

\author{
Stelios Kerassidis* \\ School of Health Sciences, University of Crete, Greece \\ Received March 15, 2009; accepted March 30, 2009
}

\begin{abstract}
In the context of the research on the antecedents of palmar hyperhidrosis, we compared hyperhidrotic in palm and normal individuals on parameters that could be related to excessive sweating. We performed neurological examination, general hematological tests, and measurements of blood levels of thyroid hormones FT3, FT4, TSH, glucose, and electrolytes Na, $\mathrm{K}, \mathrm{P}, \mathrm{Ca}, \mathrm{Mg}$. The participants also filled in the Eysenck Personality, as well as a laboratory constructed Questionnaire. Significantly more hyperhidrotic than normal individuals had a close relative suffering from palmar hyperhidrosis. In addition, hyperhidrotics scored significantly higher in Neuroticism scale of Eysenck Personality Questionnaire. No indication of neurological damage, dysautonomy, hematological abnormality, electrolytic or glucose imbalance, and thyroid gland dysfunction was found for hyperhidrotics. The findings of the present work as well as the ones of previous works suggest that palmar hyperhidrosis cannot be attributed to pathological or anatomical features, it is possibly caused by personality traits, while a genetic predisposition may also contribute.
\end{abstract}

Key words: Hyperhidrotic; Palmar sweating; Sweat glands

\section{INTRODUCTION}

Palmar hyperhidrosis refers to excessive local sweating on the palms of the hands. Probably because this sweating is an every day life problem with many social but without life threatening consequences, the publications on palmar hyperhidrosis focus mainly on the treatment and not on the pathophysiology of the disorder. Since the reviews by Sato et al. (1989) on the disorders of sweat glands, by Lerer (1977), Lerer et al. (1988) on the psychological aspects of palmar hyperhidrosis, by Fotopoulos \& Sunderland (1978) and Eisenach et al. (2005) on the treatment of psychophysiological disorders, where the lack of publications on the etiology of palmar hyperhidrosis was underlined, very little work has been done. What we already know is that (i) the sweat glands of hyperhidrotics do not defer from those of normal sweating individuals and that they are not more intensively activated after acetylcholine injection (Sato \& Sato, 1983) (ii) palmar hyperhidrosis is not controlled by a thermoregulatory mechanism (Kerassidis, 1994), (iii) excessive sweating of hyperhidrotics in palms could not be considered as defensive response after stimulation of startling kind (Kerassidis \& Charistou, 2000), (iv) Heart Rate Variability Analysis did not reveal statistical significant differences between hyperhi-

\footnotetext{
*E-mail: kerassidis@nlg.gr
}

drotic and normal individuals, sympathetic overactivation or any other autonomic imbalance for hyperhidrotics (Kerassidis \& Kohiadiakis, 1998), (v) hyperhidrotics have not a higher density of sweat glands on palm in relation to normal individuals (unpublished data). We have to mention that we consider the only anatomical suggestion of Shih et al. (1983), concerning the etiology of palmar hyperhidrosis, as incorrect. The authors suggest that palmar hyperhidrotics have an overfunctioning of sympathetic nervous fibers passing through the 2 and 3 thoracic ganglia (T2,3), which play an important role in the modulation of autonomic function elsewhere. But (i) the Heart Rate Variability Analysis did not confirm this suggestion (as it is mentioned above), (ii) hyperhidrotics usually express plantar hyperhidrosis too, but there is not any suggestion for a simultaneous over-functioning of the sympathetic nervous fibers passing through the 1 and 2 lumbar $(\mathrm{L} 1,2)$ ganglia, which innervate the sweat glands of the soles (iii) palmar hyperhidrotics, who underwent surgical treatment (bilateral upper dorsal sympathectomy of T2,3 ganglia), suffered postoperatively from plantar hyperhidrosis (Bogokowsky et al., 1983), a fact which suggests that there is probably a central and not a local anatomic reason for the palmoplantar hyperhidrosis and 
(iv) if this over-function of the sympathetic fibers plays an important role in the modulation of autonomic function elsewhere, as the authors claimed, palmar hyperhidrotics must express forearm or chest hyperhidrosis too, since the sweat glands of the forearm and chest receive innervation from the same fibers passing through T2,3 sympathetic ganglia. But this does not happened. In the present work we compare hyperhidrotic and normal individuals on any of the remaining unexplored parameters that could be related to excessive sweating. Laboratory and clinical tests, including the following, were performed: 1. standard neurological examination, in order to check for dysautonomy or neurological damage, 2. general hematological, investigating for a possible association of palmar hyperhidrosis with a blood abnormality, 3. measurement of blood levels of hormones FT3, FT4, TSH, investigating for a possible association of palmar hyperhidrosis with thyroid gland over-function (which can cause excessive sweating by accelerating the metabolic process), 4. measurement of blood glucose levels, in order to check for a possible association of palmar hyperhidrosis with hypoglycemia (and to reveal diabetic individuals), 5. measurement of blood levels of $\mathrm{Na}, \mathrm{K}, \mathrm{P}, \mathrm{Ca}, \mathrm{Mg}$, investigating for a possible association of palmar hyperhidrosis with electrolyte imbalance or parathyroidism. Edelberg (1973) suggested that skin may have a role as an accessory kidney in control of water and electrolyte balance. In order to examine whether personality traits could differentiate hyperhidrotics from normal individuals filling in the Eysenck Personality Questionnaire (EPQ) and filling in a laboratory constructed questionnaire, in order to examine whether some habits, diseases or heredity could differentiate hyperhidrotics from normal individuals (Kerassidis, 1994; Kerassidis \& Charistou, 2000).

\section{MATERIAL AND METHODS}

Participants: Forty individuals participated in the tests. Twenty individuals were hyperhidrotic in their palms (and, usually, in their soles as well), whereas the remaining 20 individuals were normal in terms of palmar sweating. Twelve females and 8 males were included in each group. The mean age of the hyperhidrotic females was 31.4 and of the normal females was 27.3 years. The mean age of the hyperhidrotic males was 35 and of the normal males was 34.3 years. The mean body weight of the hyperhidrotic females was $60.3(50-73) \mathrm{kg}$ and of the normal females was $56.4(45-70) \mathrm{kg}$. The mean body weight of the hyperhidrotic males was 75.6 (62$87) \mathrm{kg}$ and of the normal males was $76.2(62-102) \mathrm{kg}$. The educational level of hyperhidrotics/normal was $11 / 12$ higher education, 3/4 students, and $6 / 4$ secondary-lower. No individual of our study was alcohol or drug addicted, or under medication. Individuals with palmar hyperhidrosis were recruited by the local mass media. Among those who volunteered to participate, six persons who manifested excessive sweating all over the body, or predominantly in other areas of the body, like the forehead and the armpits, were excluded of the research. Normal palmar sweating individuals were selected by the authors, among people who reported never having suffered from excessive palmar sweating. In order to confirm the participants' estimation of the degree of their palmar sweating, a sweat collecting plaster (waterproof construction) was attached to each participant's left palm, while she/he was writing for two minutes on a paper with the right hand. The mean weight of the sweat collected from the palm of hyperhidrotics was $20 \mathrm{mg}$ (S.D. 14.9) and from that of normal individuals $3.1 \mathrm{mg}$ (S.D. 2.3). Out of the 42 individuals who were subjected to this confirmation test, one from the hyperhidrotic group had $4 \mathrm{mg}$ palmar sweat and one from the normal group had $12 \mathrm{mg}$ palmar sweat. The scores of these two persons were excluded from the final evaluation.

Design and materials: The participants underwent a standard neurological examination and were subjected to the laboratory blood tests: general hematological, blood glucose concentration as well as blood levels of thyroid hormones (FT3, FT4, TSH) and electrolytes $(\mathrm{Na}, \mathrm{K}, \mathrm{P}, \mathrm{Ca}, \mathrm{Mg})$. The participants answered many kinds of questions including: alcohol and drug consuming, medication, habits of sleep, state of digestive system, feelings of palpitation, suffocation, dizziness, skin problems, diseases in general, the time of the first appearance of hyperhidrosis, its expression in every day life, relatives suffering from hyperhidrosis etc. The Greek translation of the Eysenck Personality Questionnaire- EPQ (Demetrioy, 1986), comprising 84 questions and 4 scales (Neuroticism, Psychotism, Extroversion and Lying), was completed after the end of the tests.

Statistics: Two factor (sex and hyperhidrosis) analysis of variance (ANOVA) was used for comparisons between hyperhidrotic and normal individuals and between females and males in all tests.

\section{RESULTS}

Eight out of the 20 hyperhidrotics and 1 out of the 20 normal individuals declared that they had at least one close relative suffering from palmar or plantar hyperhidrosis. This difference is significant, $\mathrm{F}=6.40, \mathrm{p}<0.004$. No other systematic difference between hyperhidrotic and normal individuals was revealed by the laboratory constructed questionnaire. The neurological examination did not reveal any neurological damage or dysautonomy to any individual of both groups. No significant differences were found between hyperhidrotic and normal individuals in the general blood test, or in blood levels of thyroid hormones, glucose, or electrolytes. However, hyperhidrotics displayed higher scores than normal individuals (13.35 / 9.75) in EPQ Neuroticism scale 
$(\mathrm{F}=6.352, \mathrm{p}<0.01)$. There was no significant difference in any of the 3 other EPQ scales (Psychotism, Extroversion and Lying). Differences between females and males were not significant.

\section{DISCUSSION}

It was found that significantly more hyperhidrotics than normal individuals had a close relative suffering from palmar hyperhidrosis. In addition, hyperhidrotics displayed higher scores than normal individuals in Neuroticism scale of EPQ. There was no indication of neurological damage, dysautonomy, hematological abnormality, electrolytic or blood glucose imbalance, and thyroid gland dysfunction for hyperhidrotics. The finding that significantly more palmar hyperhidrotics than normal individuals have a close relative suffering from palmar hyperhidrosis $(40 \%$ of hyperhidrotics, and only $5 \%$ of normal individuals) suggests that a genetic predisposition to excessive palmar sweating may exist. Previous works, like those of Kwon et al. (1998), and James et al. (1987) are in line with this suggestion. Especially, James et al. described a family with hereditary hyperhidrosis and they suggest that the inheritance pattern is autosomal dominant. Although an inheritance pattern of this kind cannot be accepted as the general transmission way of palmar hyperhidrosis, from the parent to the offspring, and although there is no idea concerning the nature of the transferred characteristics which could cause palmar hyperhidrosis to offspring, the evidences indicate that a genetic factor may contribute to the disorder.The higher score of hyperhidrotics than that of normal individuals in Neuroticism scale of EPQ is not the only finding leading the search of the etiology of palmar hyperhidrosis in personality characteristics. In another work (Kerassidis \& Bitzaraki, 1997), we have also found higher scores for hyperhidrotics than normal individuals in Psychotism, Depression and Social Introversion of Minnesota Multifasic Personality Inventory (MMPI). In line, the findings of Lerer, et al. (1977) and of Lerer and Jacobowitz (1981) suggest that hyperhidrotic individuals are characterized by lower overall ability to cope with stress and a strong proclivity to avoid problems. The discrepancy between these findings and that of Kwon et al. (1988) is rather deceptive. Kwon et al. found that their hyperhidrotic group (on palms, soles, and axillae) scored within the Korean normal ranges of EPQ and concluded that there is no evidence of abnormal personality features. Our hyperhidrotic group also scored within the Greek normal ranges of EPQ. However Kwon et al. did not use a normal matched group for comparisons. So, they found that hyperhidrotics are not psychiatric patients but they fail to reveal any difference, concerning personality characteristics, between hyperhidrotics and a matched normal group. The statistically significant higher score on EPQ Neuroticism scale of hyperhidrotic in relation to the score of matched normal individuals, in our work, do not put hyperhidrotics in a psychiatric patients group, but probably indicate that hyperhidrotics in relation to normal individuals are more anxious, with more intense emotional reactions, and a proclivity in psychosomatic disorders (Eysenck \& Eysenck, 1975). It is somehow like a group of workers in a dangerous employment to present statistically significant higher blood pressure values than their colleagues in less dangerous employment, without the test group to be hypertensive. We have to notice that the revelation of personality differences between hyperhidrotic and normal individuals did not automatically mean that personality traits are the antecedents of palmar hyperhidrosis. Individuals who suffer from palmar hyperhidrosis could develop neurotic personality characteristics as a consequence of the hyperhidrosis condition, since hyperhidrotic patients may suffer continued embarrassment and disruption of their school, work and social lives (James et al., 1987). Although the influence of hyperhidrosis disorder on the personality of hyperhidrotics may exist, we have indications that palmar hyperhidrosis can be caused from psychological reasons. Some hyperhidrotics who participated to our research declared that they started to exhibit excessive palmar sweating when they faced a serious stressor. We have also seen cases of transient palmar hyperhidrosis during psychological stressed periods of an individual's life (a colleague of the laboratory of pharmacology presented palmar hyperhidrosis during certain months when he has to defend his thesis, to be engaged, and to prepare to enlist in the army). The fact that palmar hyperhidrosis can be caused by psychological reasons indicates that personality characteristics may be the antecedent of the disorder. Of course, the exact mechanism remains to be elucidated. We think that the revelation of this mechanism must be the future task of the investigation on the antecedents of palmar hyperhidrosis. Taken into account the findings of the present work as well as those of the previous works mentioned in the introduction section, we concluded that palmar hyperhidrosis cannot be attributed to pathological or anatomical features; it is possibly caused by personality traits, while a genetic predisposition may also contribute.

\section{Acknowledgement \\ The present work was supported by the Laboratories of Clinical Chemistry and Biochemistry as well as the Nuclear Medicine of the University Hospital of Crete.}

\section{REFERENCES}

Bogokowsky, H., Slutzki, S., Bacalu, L., Abramsohn, R., \& Negri, M. (1983). Surgical treatment of primary hyperhidrosis. A report of 42 cases. Archives of Surgery, 18, 1065-7. 
Demetrioy, E.C. (1986). The Eysenck Personality Questionnaire (EPQ): The validity of the Greek, adult and junior, visi-

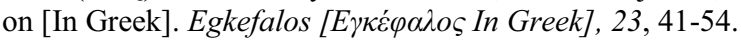

Edelberg, R. (1973). Mechanisms of electrodermal adaptations for locomotion, manipulation, or defense. In: E. Stellar \& J.M. Sprague (Eds.), Progress in Physiological psychology, pp. 155-209. New York: Academic Press.

Eisenach, J.H., Atkinson, J.L., \& Fealey, R.D. (2005). Hyperhidrosis: evolving therapies for a well-established phenomenon. Mayo Clinic Proceedings, 80, 657-666.

Eysenck, H.J., \& Eysenck, S.B.G. (1975). Manual of the EPQ (Personality Questionnaire). London: Hodder and Stoughton. Fotopoulos, S.S., \& Sunderland WP. (1978). Biofeedback in the treatment of psychophysiologic disorders. Biofeedback and Self-regulation, 3, 331-361.

James, W., Schoomaker, E., \& Rodman C. (1987). Emotional Eccrine Sweating. Archives of Dermatology, 123, 925-929.

Kerassidis, S. (1994). Is palmar and plantar sweating thermoregulatory? Acta Physiologica Scandinavica, 152, 259-263.

15. Kerassidis, S, \& Bitzaraki, K. (1997). Personality traits of

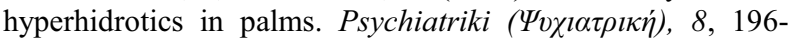
202.

Kerassidis, S., \& Charistou, A. (2000). Nonresponders among hyperhidrotics. Biological Psychology, 52, 85-90

Kerassidis, S, \& Kohiadiakis, G. (1998). Heart Rate Variability Analysis in hyperhidrotics in palms [In Greek]. Hellenic Journal of Cardiology, 39, 474-478.

Kwon, O.S., Kim, B.S., Cho, K.H., Kwon, J.S., Shin, M.S., Youn, J.I., \& Chung, J.H. (1998). Essential hyperhydrosis: no evidence of abnormal personality features. Clinical and Experimental Dermatology, 23, 45-6.

Lerer, B. (1977). Hyperhidrosis: A review of its psychological aspects. Psychosomatics, 18, 28-31.

Lerer, B., Jacobowitz, J., \& Wahba, A. (1980). Personality features in essential hyperhidrosis. International Journal of Psychiatry in Medicine, 10, 59-67.

Lerer, B., \& Jacobowitz, J. (1981). Treatment of essential hyperhidrosis by psychotherapy. Psychosomatics, 22, 536538.

Sato, K., Kang, W., Saga, K., \& Sato, K.T. (1989). Biology of sweat glands and their disorders. I. Disorders of sweat gland function. Journal of the American Academy of Dermatology, 20, 713-726.

Sato, K.T., \& Sato, F. (1983). Individual variations in structure and function of human eccrine sweat gland. American Journal Physiology, 243, R203-R208.

Shih, C.J., Wu, J.J., \& Lin, M.T. (1983). Autonomic dysfunction in palmar hyperhidrosis. Journal of the Autonomic Nervous System, 8, 33-43. 